\title{
Research Article \\ Effect of Contact Conditions of Torsional Wave Elastographic Probe on Human Cervix
}

\author{
G. Rus $(\mathbb{D}$, M. Riveiro, and F. S. Molina \\ Ultrasonics Group TEP-959, Biomechanics Group TEC-12 of the Biosanitary Research Institute IBS, \\ MNat Excellence Scientific Unit UCE.PP2017.03, NDE Lab, Department of Structural Mechanics, University of Granada, Spain
}

Correspondence should be addressed to G. Rus; grus@ugr.es

Received 14 September 2018; Revised 1 November 2018; Accepted 18 November 2018; Published 29 November 2018

Academic Editor: Alessandro Lo Schiavo

Copyright (C) 2018 G. Rus et al. This is an open access article distributed under the Creative Commons Attribution License, which permits unrestricted use, distribution, and reproduction in any medium, provided the original work is properly cited.

\begin{abstract}
There is a challenge in characterizing the soft tissue mechanical functionality in cylindrical geometries by current elastography techniques applied to small organs. Torsional waves are a type of shear elastic waves that propagate through soft tissue radially and in depth in a curled geometry, ideally suited to explore structures such as the cervix. Here, a sensor based on a novel arrangement of concentric sandwiches of piezo- and electromechanical elements is prototyped and tested to quantitatively assess stiffness in human cervix. The purpose is to determine the robustness of a torsional wave elastography (TWE) sensor prototype to quantify cervical stiffness, as a first step to validate a reliable measurement protocol using TWE. An array of tests designed to validate a set of hypothesis about the contact conditions were performed on up to 3 different nonpregnant voluntary women. The mechanical properties were reconstructed from the recorded TWE signals, and the outcomes were statistically analyzed to validate the contact condition hypothesis. The results suggest that, although future tests need to be performed to fully assess the repeatability and quality of the measurements, some characteristics of the measurement protocol become clear; in particular: (1) the use of speculum is recommended since it not only stabilizes the measurements, but also corrects and stabilizes the orientation of the cervical neck, and allows to visually verify the positioning and alignment of the probe, while not significantly increasing discomfort to women during this test; (2) the use of lubricant gel has no significant effect on the measurement quality; (3) $1 \mathrm{~cm}$ off-centering from external cervical os is not acceptable; (4) a range of applied force between 500-2000 N does not significantly vary measurement signal quality, but the reconstructed value of the shear modulus does vary, probably due to its constitutive nonlinearity; (5) breathing movements affects measurements, but short duration of TWE pulse $(0.3 \mathrm{~s})$ allows to take measurements in apnea.
\end{abstract}

\section{Introduction}

The structural microarchitecture of soft tissue is recently catching attention among the biomechanics community, and it is gaining interest for clinical diagnosis in a broad spectrum of medical specialties, since quantitative measurement of cervical elasticity by shear waves in vivo constitutes a new diagnostic principle that only recently is being proposed [15]. This concept is first being applied to early diagnosing birth and labor disorders, such as premature ripening of the cervix, delivery induction failures, etc.

In the last years, elastography has been proposed by many authors to evaluate cervical stiffness/softness $[1-3,6,7]$, as there is no clinical tool for the quantitative and objective assessment of the cervical biomechanical state during pregnancy [4]. The World Health Organization (WHO) estimates that in 2017 approximately 15 million babies will be born preterm ( $<37$ weeks of gestation), this is, a rate above 1 in 10 newborns [8]. Worldwide, complications of preterm birth have supplanted pneumonia as the primary cause of child mortality for children under five years of age $[8,9]$. The problem is not isolated to the developing countries, as preterm birth rates are rising in most countries in Europe [10]. While some progress is being made in identifying cultural and/or socioeconomic risk factors of preterm birth, there is a considerable proportion of unpredictable spontaneous preterm delivery, and the biology of cervical ripening that leads to birth remains poorly understood.

To objectively quantify cervical stiffness, a TWE sensor prototype was designed, prototyped and was the object of the present test for measurement robustness. Unlike commercial elastography, torsional wave is shear elastic waves 
that propagate through soft tissue radially and in depth in a curled geometry, perfectly suited to the small size and specific cylindrical shape of the cervix, which challenge ARFI-based elastography [11]. The TWE sensor is based on a novel arrangement of concentric sandwiches of piezo- and electromechanical elements, which enables the precise interrogation of soft tissue mechanical functionality in cylindrical geometries.

The purpose of this work is to evaluate the robustness of the mechanical characterization of the cervix through the TWE probe, specifically against some relevant contact conditions. By robustness we define the maintenance of measurement signals quality and consistency against variations in a set of examination procedures and errors during the application of the clinical probe operation. The specific objectives are

(O1) To verify the positioning, orientation and alignment of the probe respect to the cervix.

(O2) To determine the need of speculum.

(O3) To determine the need of lubricant gel.

(O4) To validate the robustness against probe cervical os off-centering.

(O5) To analyze the effect of probe applied force on the cervix within a soft force range.

To this end, an array of tests designed to validate a set of hypothesis about the contact conditions were performed on up to 3 different nonpregnant voluntary women. The mechanical properties were reconstructed from the recorded TWE signals, and the outcomes were statistically analyzed to validate the contact condition hypothesis.

\section{Methods}

The endovaginal examination with the TWE probe was simultaneously guided by an abdominal sagittal ecography to assess cervical alignment to endocervical canal (to analyze objective O1), and to confirm the probe position on the cervix using or not speculum (to analyze objective O2). The probe was positioned in contact with the cervical lips, centered at the os. The torsional waves propagate in depth and radially interrogating the compound dermal, internal stromatic connective tissue, therefore not excluding mucus, epithelium, and crypts, which are a priori expected to be a potential explanation for measurement complications to be tested. An examination series was performed with and without lubricant gel on the probe (to analyze objective $\mathrm{O} 3$ ). Measurements in external cervical os against other regions of the cervix (anterior, posterior and lateral lips) were obtained (to analyze objective O4). The operators applied first a normal force and second a soft force on the cervix, in a range of 500$2000 \mathrm{~N}$ (equivalent to 50-200 g). A training for the operator was required to apply a uniform force, within an error of subjective perception around $\pm 20 \%$ which was estimated through testing probe force in a balance (to analyze objective O5). The variation between measurements taken at the start and the end of the examination protocol in each woman was calculated. The protocol sequence was repeated twice by each

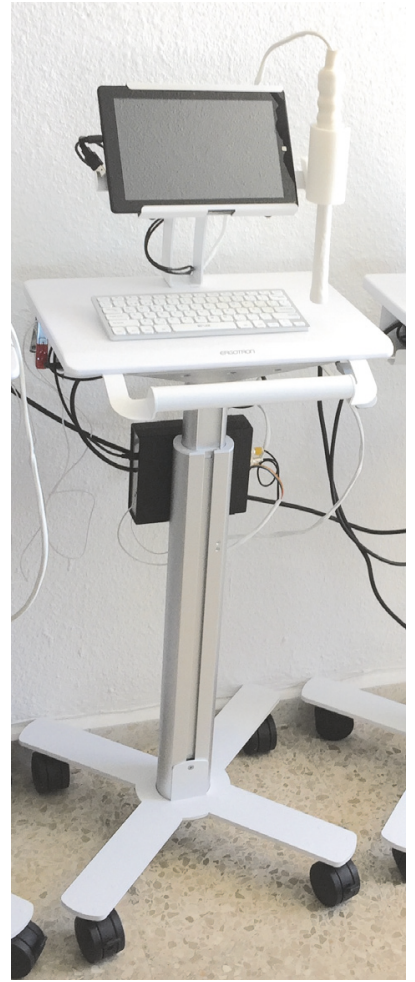

FIGURE 1: In-lab designed and prototype torsional wave elastography probe.

operator. Breathing and postural movements of the subjects were recorded during measurement.

2.1. Torsional Wave Elastography. Elastography was performed using an in-lab designed and prototyped probe capable of generating, receiving and analysing torsional waves free of spurious compression waves, as well as sensing them at safe radiation energies. The proof of concept device (Figure 1) is composed of three parts; sensor, generation/receiver electronic system, and the interface software. The proposed sensor concept is based on (1) a transmitting disc with a sandwich design combining piezoceramic or electromechanical elements to control impedance and inertia, resonant frequency and energy transmission to reduce dilatational waves, and a Faraday shield to control electronic cross-talk. The receiving part consists of a (2) crown ring sandwich with a converse design to the transmitter, encapsulated in (3) a casing with geometrical and material selection to control the mechanical cross-talk. This configuration eliminates the masking $\mathrm{p}$-waves that systematically arise at the boundaries of the regular contact or comb transducers. Further technical details are processing for reconstruction of mechanical parameters are covered by patents [12-14] and previous publications by the authors $[15,16]$. From the reconstructed shear wave velocity $c_{s}$, it is possible to compute a shear elasticity under the assumption of a purely elastic and incompressible medium, for which the shear modulus $\mu$ can be written as

$$
\mu=\rho c_{s}^{2}
$$




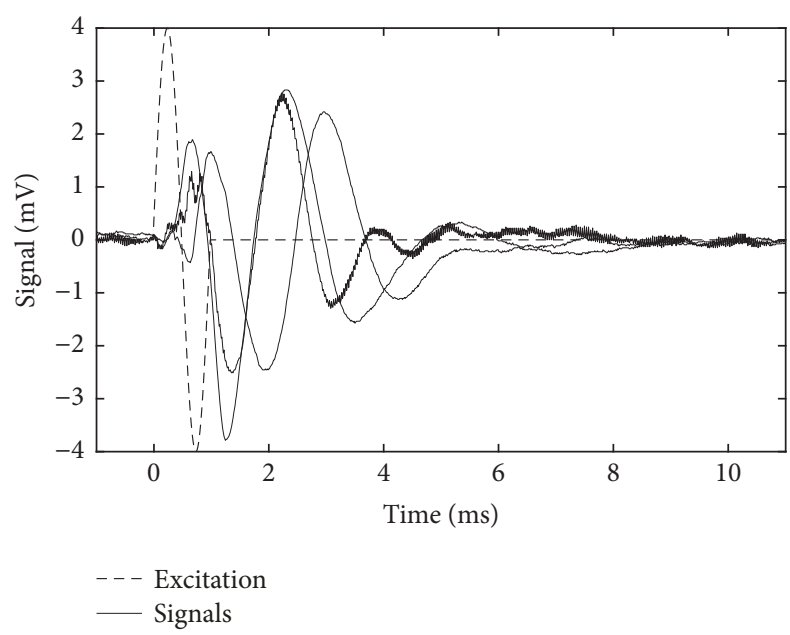

FIGURE 2: Illustrative example of three transmitted and received $1 \mathrm{kHz}$ signals.

where $\rho$ is the density of the medium. In order to describe soft tissue elasticity, the usual parameter is Young's modulus, $E=\mu(3 \lambda+2 \mu) /(\lambda+\mu)$. In macroscopic and homogeneous media $\lambda$ is $10^{4} \sim 10^{6}$ times larger than $\mu$, thus a good and an usual approximation of $E$ is: $E=3 \mu$. Thus, Young's modulus can then deduced from the shear wave velocity as

$$
E=3 \rho c_{s}^{2}
$$

The propagated torsional wave is a burst configured as a 1-cycle sinusoid of frequency $f$ between 0.5 and $1.5 \mathrm{kHz}$ with $10 \times$ averaging. An example of the transmitted and received signal is shown in Figure 2.

2.2. Settings and Ethical Issues. The study was conducted in the Fetal Medicine Unit at the Universitary Hospital Complex of Granada, Spain, in March 2017. The prototype of the probe and the analysis of the data were performed at the Non-Destructive Laboratory (University of Granada). The study was designed according to the Declaration of Helsinki, with the approval of the 'Comité de Ética de la Investigación Biomédica de Granada, the Comité de Ética de la Universidad de Granada,' and the 'Comisión de Ética e Investigación Sanitaria del Hospital Universitario San Cecilio de Granada.'

2.3. Population. Eligible participants were non-pregnant, $>18$ years old women. Exclusion criteria were cervical surgery (e.g., conization), premalignant or malignant cervical changes, and menopause. All the elegible women agreed to participate and gave written informed consent. Datasets were anonymized.

2.4. Outcome Measures. The outcomes to compare the set of measurements in the protocol were cervical stiffness $(G)$, estimated through the velocity of signal, and signal quality (Q).

2.5. Statistical Analysis. To compare the effects of using a speculum, a lubricant gel, a range of pressure, and the probe off-centering from cervical os on the measurements, the ANOVA test was used. Stiffness was normalized to a reference subject (A) within women. The p-values for all hypothesis were 2-sided and a statistical significance was considered at $\mathrm{p}<0.05$. Data were analysed using the MATLAB [17], particularly packages ttest 2 and anoval [18].

\section{Results}

A total of 3 women aged 27-39 years were recruited for examination through TWE technique. They were one primiparous, one multiparous, and one nulliparous woman. Obstetrical formulae of term-birth/preterm-birth $(<37$ weeks)/Abortus/Living children (TPAL) were T1P0A2L1, T2P0A1L2, and T0P0A0L0, respectively.

The measurements protocol through TWE probe could not be totally applied in some of the participants. A time interval of 30 minutes was considered to test variation between outcomes of measurements from the start to the end of the protocol application in one woman.

ANOVA analysis results for the tested hypothesis are shown in Table 1 and illustrated in Figure 3, including the specification of the hypothesis to be tested, the number $N$ of patients, and the number of operators. p-value and t-stat are provided, as well as the summary of the reconstructed stiffness modulus $G$ and the quality estimate $Q$.

The limitation of the small population ( 3 women) theoretically increases the risk of type-II error, but due to the multiplicity of measurements taken intraoperator and, as the p-values indicate, most of the hypotheses are tested with statistical significance, overcoming the limitation for the goals of this study. In the next studies, a larger population will be required for inter-operator repeatability and other tests.

\section{Conclusions}

The statistical analysis of the tested hypothesis about the contact conditions allow us to draw a number of conclusions, detailed below, which provide initial hints of the measurement protocol to be applied by the clinician to operate the present TWE probe. However, further hypothesis needs to be tested, which are the goal of a future work, to assess the feasibility of objectively quantifying cervical stiffness through a TWE sensor prototype with repeatability and reliability, upon a measurement protocol to be designed.

(i) The use of speculum is recommended during TWE examination since it corrects and stabilizes the orientation of the cervical neck, and allows to visually verify the positioning and alignment of the probe, as supported with simultaneous abdominal echography. Although the use of speculum becomes a training requirement for the operator, the speculum does not significantly increase discomfort to women during this test.

(ii) The use of lubricant gel has no significant effect on the measurement quality, and it is therefore proposed to be optional. 


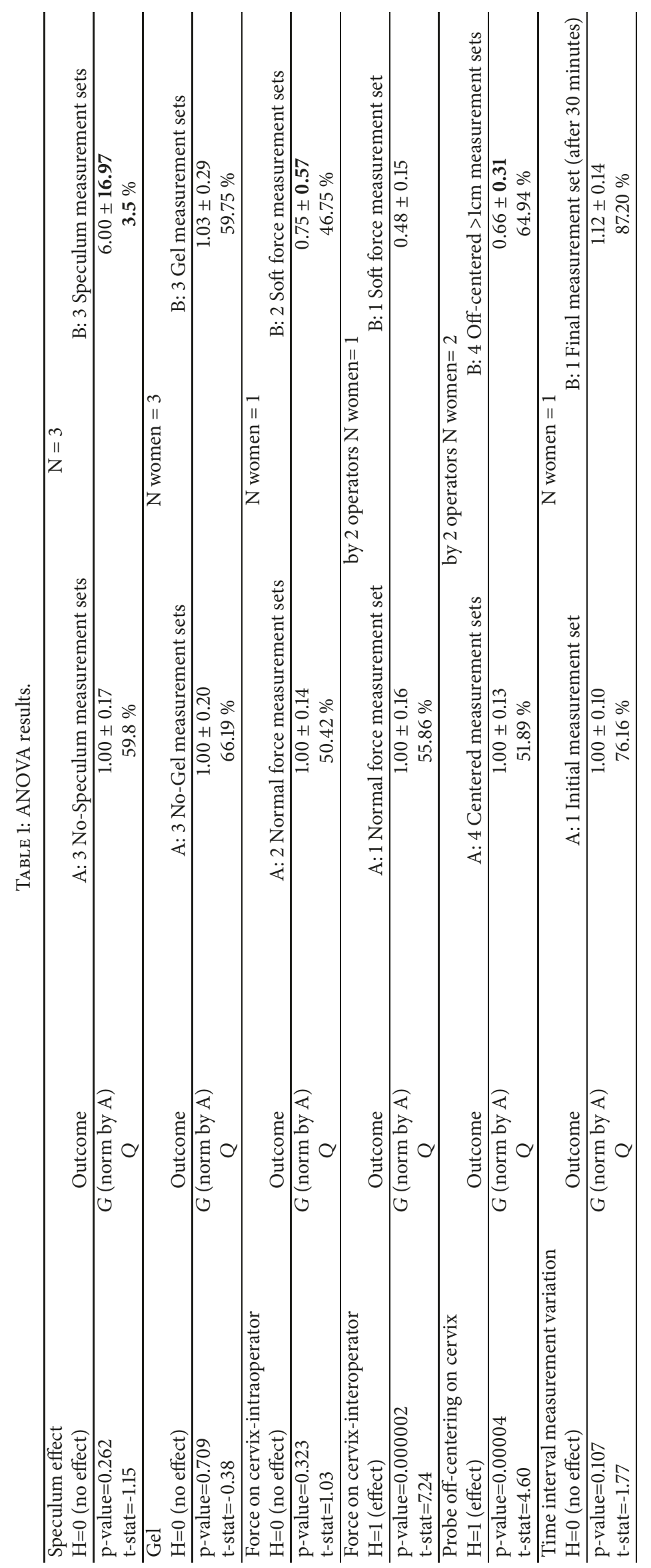




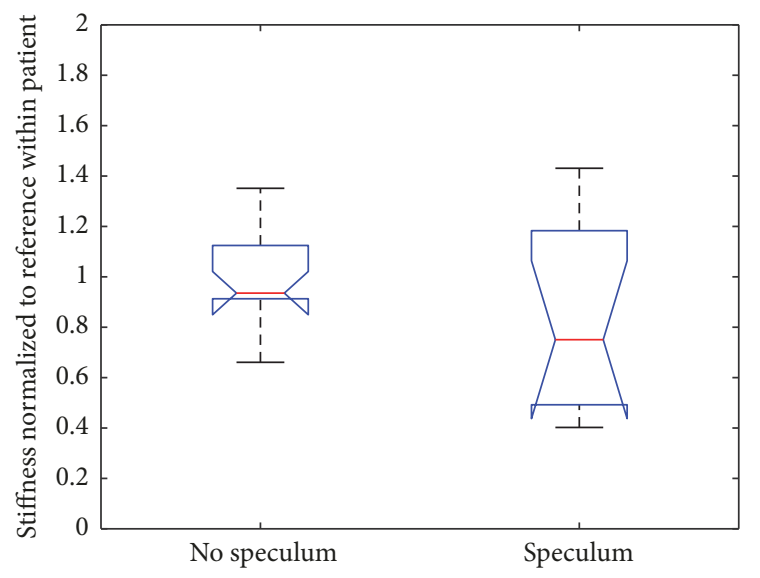

(a)

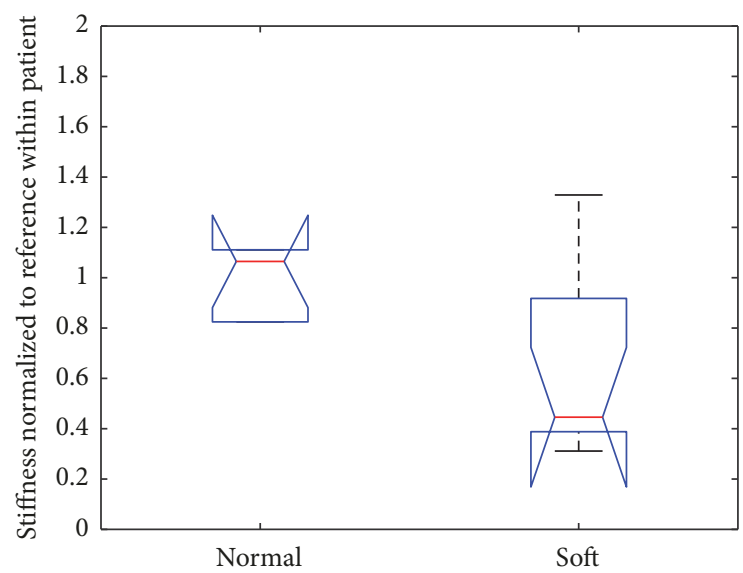

(c)

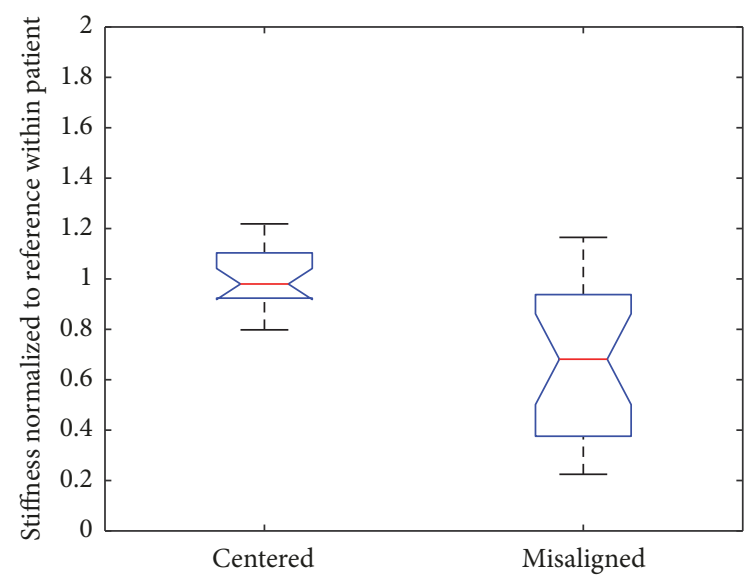

(e)

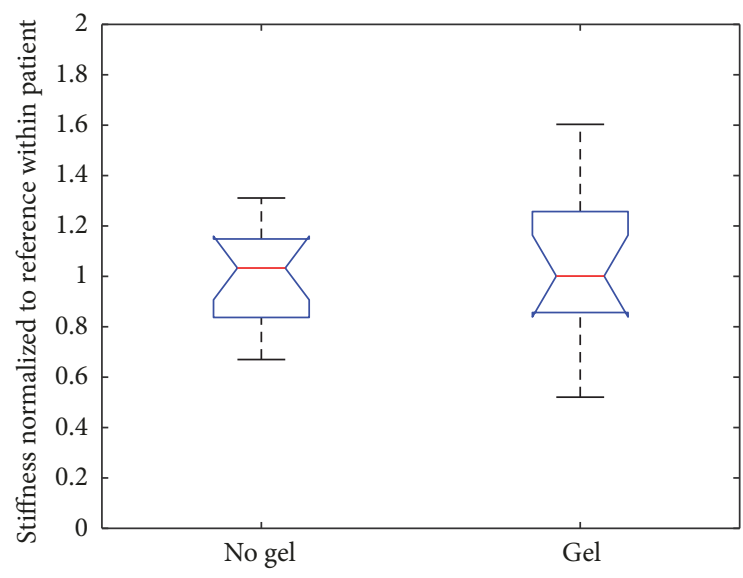

(b)

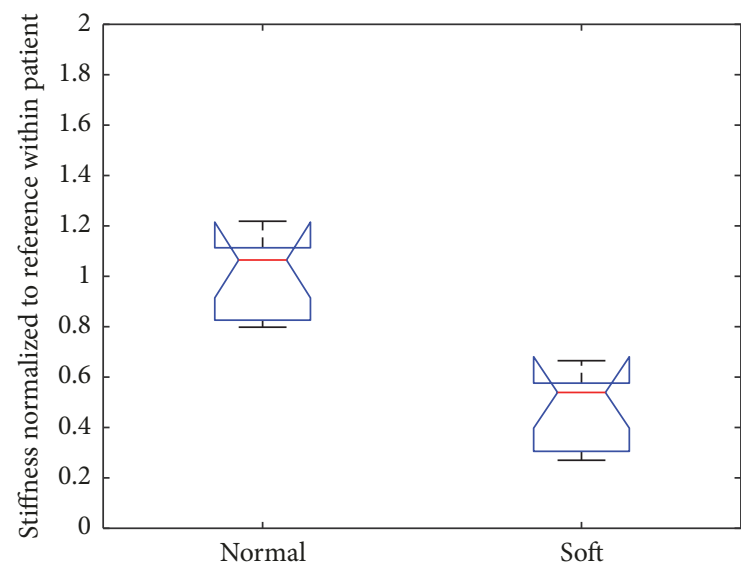

(d)

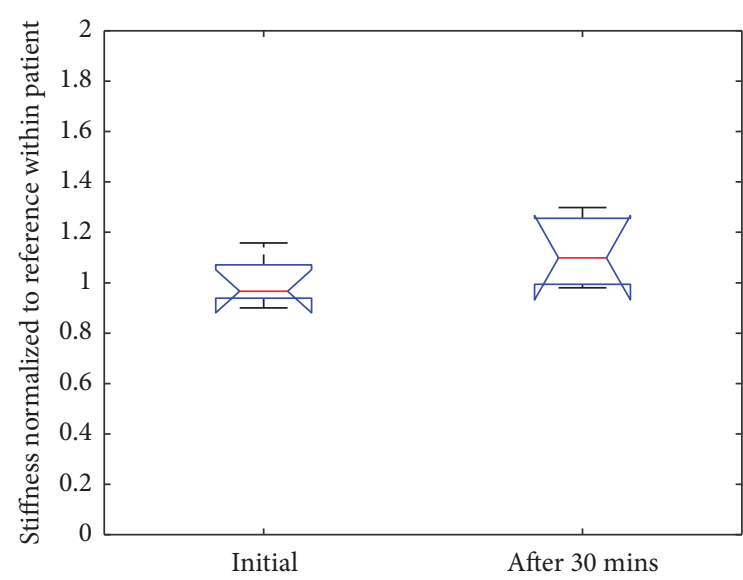

(f)

FIgURE 3: ANOVA box plots for testing each hypothesis indicated on the left vertical axis.

(iii) A threshold of $1 \mathrm{~cm}$ off-centering from external cervical os divides measurements into stable or unstable and therefore repeatable or non-repeatable. Therefore, such a misalignment is not acceptable.

(iv) A range of applied force between 500-2000 N does not significantly vary measurement signal quality.
However, the reconstructed value of the shear modulus does vary, probably due to the constitutive nonlinearity of the tissue.

(v) Breathing movements affects measurements, but short duration of TWE pulse $(0.3 \mathrm{~s})$ allows us to take measurements in apnea. 
(vi) Larger sample-size studies using a validated measurement protocol with a concordance test to assess interoperator and intraoperator repeatability are needed in further research.

\section{Data Availability}

The data used to support the findings of this study have not been made available because it is part of the intellectual property of the company Innitius (www.initius.com), partly covered by patent pending.

\section{Conflicts of Interest}

The authors are members of the company Innitius (https://www.innitius.com), which is in charge of the technical development of the probe used in the contribution.

\section{Acknowledgments}

The authors are grateful to Pilar Carretero, Andrea Pazos, and the volunteering patients for their collaborations. This research was supported by the Ministry of Education DPI2017-83859- R, DPI2014-51870- R, DPI2010-17065, and UNGR15-CE- 3664, Ministry of Health DTS15/00093 and PI16/00339, PI-0107- 2017, and PIN-0030- 2017, and Junta de Andaluca P11-CTS- 8089 projects.

\section{References}

[1] M. Swiatkowska-Freund and K. Preis, "Elastography of the uterine cervix: Implications for success of induction of labor," Ultrasound in Obstetrics \& Gynecology, vol. 38, no. 1, pp. 52-56, 2011.

[2] S. Pereira, A. P. Frick, L. C. Poon, A. Zamprakou, and K. H. Nicolaides, "Successful induction of labor: Prediction by preinduction cervical length, angle of progression and cervical elastography," Ultrasound in Obstetrics \& Gynecology, vol. 44, no. 4, pp. 468-475, 2014.

[3] S. Wozniak, P. Czuczwar, P. Szkodziak, P. Milart, E. Wozniakowska, and T. Paszkowski, "Elastography in predicting preterm delivery in asymptomatic, low-risk women: A prospective observational study," BMC Pregnancy and Childbirth, vol. 14, no. 1, 2014.

[4] H. Feltovich, T. J. Hall, and V. Berghella, "Beyond cervical length: Emerging technologies for assessing the pregnant cervix," American Journal of Obstetrics \& Gynecology, vol. 207, no. 5, pp. 345-354, 2012.

[5] L. C. Carlson, H. Feltovich, M. L. Palmeri, J. J. Dahl, A. Munoz del Rio, and T. J. Hall, "Estimation of shear wave speed in the human uterine cervix," Ultrasound in Obstetrics \& Gynecology, vol. 43, no. 4, pp. 452-458, 2014.

[6] M. R. Khalil, P. Thorsen, and N. Uldbjerg, "Cervical ultrasound elastography may hold potential to predict risk of preterm birth," Danish Medical Journal, vol. 60, no. 1, 2013.

[7] K. Köbbing, A. Fruscalzo, K. Hammer et al., "Quantitative Elastography of the uterine cervix as a predictor of preterm delivery," Journal of Perinatology, vol. 34, no. 10, pp. 774-780, 2014.
[8] WHO, "March of dimes, the partnership for maternal, newborn \&amp; child health, and save the children. 2012. born too soon," in The Global Action Report on Preterm Birth, C. P. Howson, M. V. Kinney, and J. E. Lawn, Eds., Geneva, 2012.

[9] WHO. Fact sheet - preterm birth. Magnetic Resonance in Medicine, 2016.

[10] J. Zeitlin et al., "Wideband mr elastography for viscoelasticity model identification," International J. of Obstetrics and Gyneco, vol. 120, pp. 1356-1365, 2013.

[11] L. Peralta, F. S. Molina, J. Melchor et al., “Transient Elastography to Assess the Cervical Ripening during Pregnancy: A Preliminary Study," Ultraschall in der Medizin / European Journal of Ultrasound (UiM/EJU), vol. 38, no. 4, pp. 395-402, 2017.

[12] G. Rus, "Procedimiento de obtencin de datos sobre la elasticidad de materiales empleando ondas de torsin, 14," PCT/ES2017/070065, 2016.

[13] G. Rus, "Dispositivo emisor de ondas ultrasnicas de torsin y transductor que lo comprende, 16," PCT/ES2016/070540, 2015.

[14] G. Rus, "Transductor ultrasnico de torsin para diagnstico tisular, 14," PCT/ES2012/070380, Article ID 070380, p. S2012, 2011.

[15] J. Melchor and G. Rus, "Torsional ultrasonic transducer computational design optimization," Ultrasonics, vol. 54, no. 7, pp. 1950-1962, 2014.

[16] A. Callejas, A. Gomez, J. Melchor et al., "Performance study of a torsional wave sensor and cervical tissue characterization," Sensors, vol. 17, no. 9, 2017.

[17] The Mathworks, Inc., Natick, Massachusetts. MATLAB version 8.5.0.197613 (R2015a), 2015.

[18] R. V. Hogg and J. Ledolter, Engineering Statistics, New York: MacMillan, 1987, Engineering Statistics. 


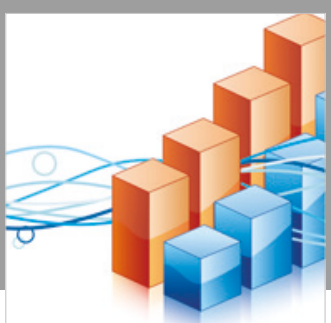

Advances in

Operations Research

\section{-n-m}
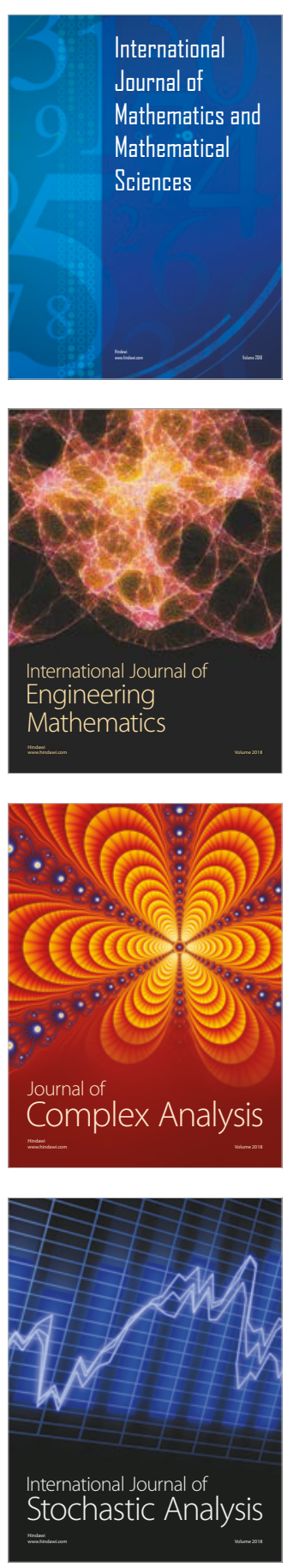
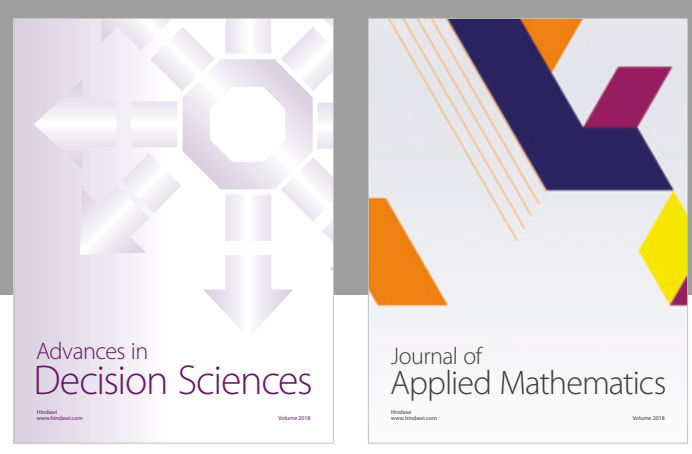

Journal of

Applied Mathematics
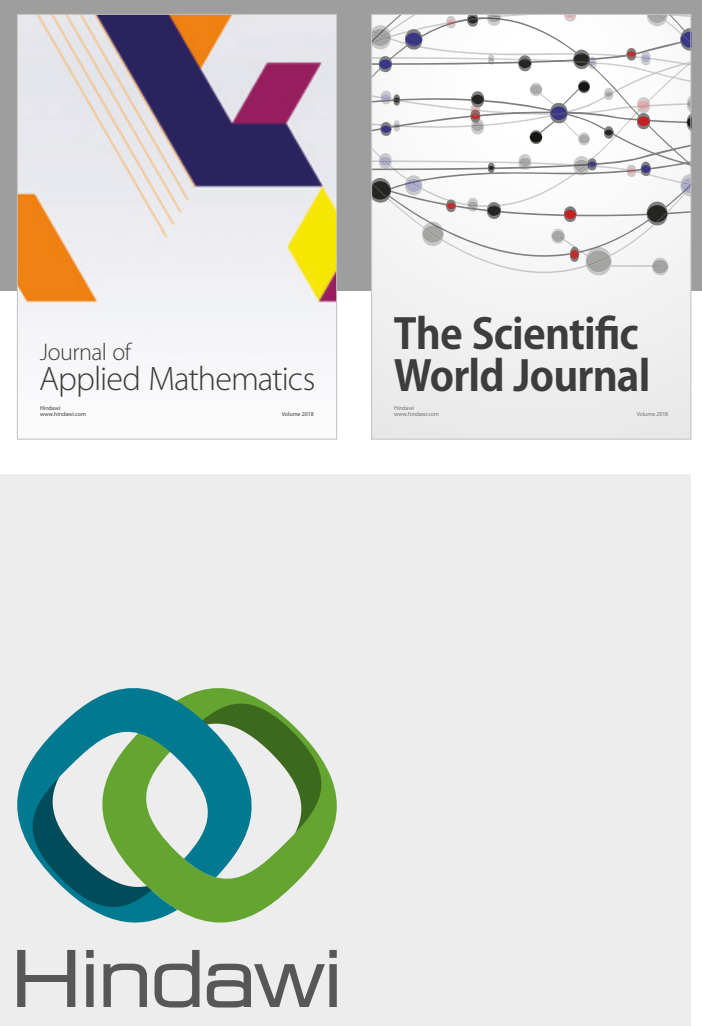

Submit your manuscripts at

www.hindawi.com

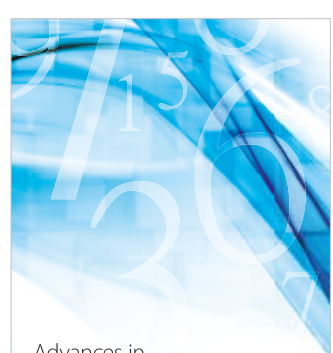

Advances in
Numerical Analysis
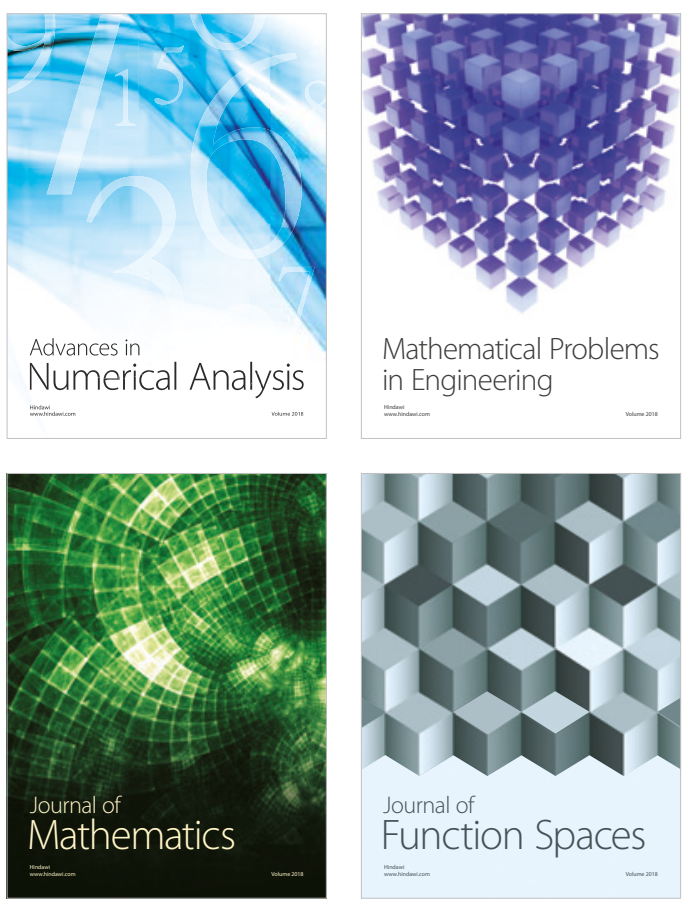

Mathematical Problems in Engineering

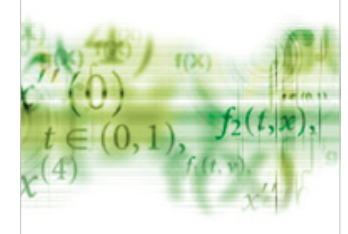

International Journal of

Differential Equations

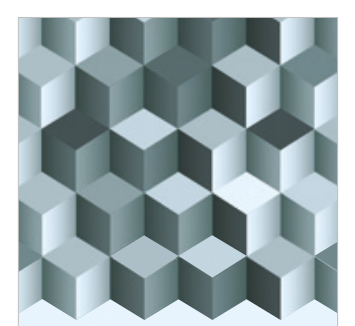

Journal of

Function Spaces

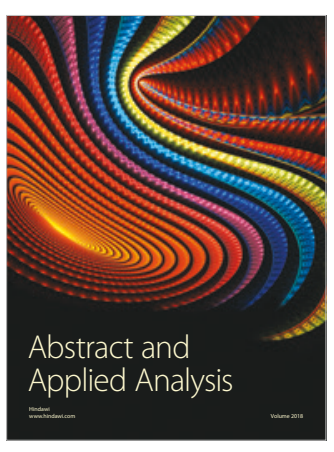

The Scientific

World Journal

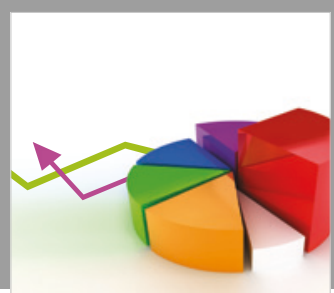

Journal of

Probability and Statistics
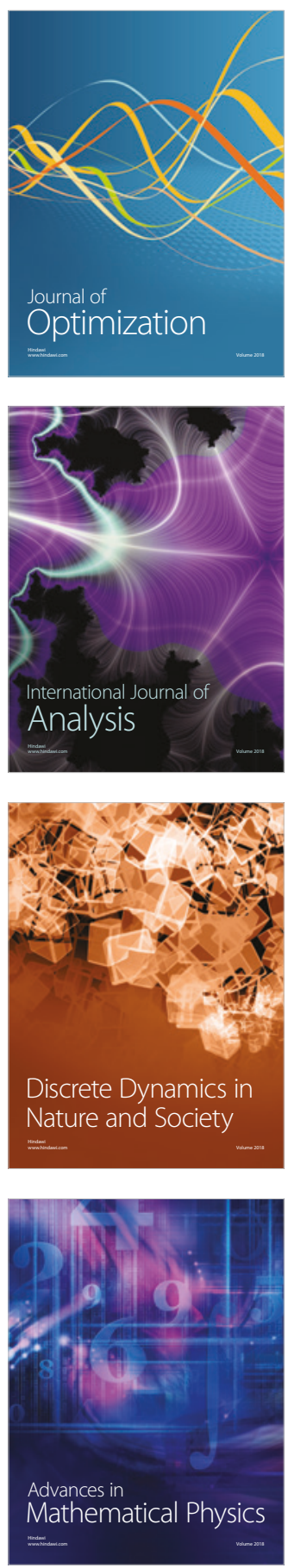